Cipango Cahiers d'études japonaises

$17 \mid 2010$

La péninsule retrouvée

\title{
Kōkan hitsudan 江關筆談
}

Conversation au fil du pinceau à Edo avec les envoyés coréens

Kōkan hitsudan 江關筆談: Brusk Talk en Edo with Korean Envoys

\section{Arai Hakuseki}

Traducteur : Vincent Grépinet

\section{(2) OpenEdition}

\section{Journals}

\section{Édition électronique}

URL : https://journals.openedition.org/cipango/1127

DOI : $10.4000 /$ cipango. 1127

ISSN : 2260-7706

Éditeur

INALCO

Édition imprimée

Date de publication : 30 juin 2010

Pagination : 89-113

ISBN : 978-2-85831-195-8

ISSN : $1164-5857$

\section{Référence électronique}

Arai Hakuseki, «Kōkan hitsudan 江關筆談》, Cipango [En ligne], 17| 2010, mis en ligne le 12 octobre 2012, consulté le 30 juin 2021. URL : http://journals.openedition.org/cipango/1127 ; DOI : https:// doi.org/10.4000/cipango. 1127

Ce document a été généré automatiquement le 30 juin 2021

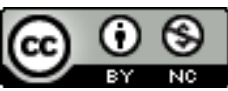

Cipango est mis à disposition selon les termes de la Licence Creative Commons Attribution - Pas d'Utilisation Commerciale 4.0 International. 


\section{Kōkan hitsudan 江關筆談}

Conversation au fil du pinceau à Edo avec les envoyés coréens

Kōkan hitsudan 江關筆談: Brusk Talk en Edo with Korean Envoys

\section{Arai Hakuseki}

Traduction : Vincent Grépinet

Nous tenons à remercier Jean-Noël Robert de l'EPHE et Philippe Che de l'Université de Provence pour leurs éclairages concernant certains passages obscurs du texte.

Le Kōkan hitsudan 江關筆談 (coréen : Kanggwan p'ildam) traduit ici est tiré du volume quatre des Cuvres complètes d'Arai Haksuseki (Arai Hakuseki zenshū 新井白石全集), p. 725-731. Yi Wŏn-sik suggère que le mot Kōkan 江關 est une métaphore qui évoque la distance entre la Corée et le Japon. Voir Chosŏn t'ongsinsa 朝鮮通信使, Minŭmsa, 1991, p. 170. Nous avons respecté, dans la mesure du possible, le système Pin yin pour transcrire les termes et les noms propres chinois, le système McCune-Reischauer pour le coréen et le système Hepburn pour le japonais.

Notes rassemblées par Cho T'ae-ŏk, sous-directeur du ministère des Fonctionnaires civils ${ }^{1}$.

Cinquième jour du onzième mois de l'année 1711.

Alors que les [envoyés] se trouvent à Edo, Hakuseki Minamoto Kimiyoshi leur rend visite et il fait les salutations d'usage en parlant de la saison.

Après avoir pris un papier et un pinceau, P'yŏngchŏn ${ }^{2}$ [commence le dialogue] en écrivant: « Nous pourrions converser directement en nous servant du pinceau. Nous nous comprendrons forcément. »

Hakuseki acquiesce poliment.

NAMgANg ${ }^{3}$ : J'ai lu dans un ouvrage de maître Ouyang Xiu ${ }^{4}$ qu'on trouve dans votre pays des textes antérieurs à la période des Qin. II semblerait qu'il en existe encore un ou deux exemplaires. Est-ce vrai ?

Hakuseki : Dans la province d'Izumo, il y a un mausolée que la population confond avec un temple ${ }^{5}$. Autrefois, j'ai entendu dire qu'on y conservait des tablettes en bambou laqué. Il s'agit vraisemblablement d'un exemplaire du Livre des Shang en chinois ancien ${ }^{6}$. 
CHŏNgP'yŏNg 7 : Ce texte doit probablement être écrit avec des signes chinois archaïques ${ }^{8}$. $Y$ a-t-il des personnes qui peuvent le déchiffrer et en a-t-on fait une copie?

Hakuseki : Nos gens le cachent car ils y tiennent beaucoup. C'est comme s'ils protégeaient quelque chose de sacré ! C'est vraiment regrettable.

P'YongchŏN : J'ai entendu dire que dans le mausolée de Xu Fu (jap. Jofuku) ${ }^{9}$ à Kumano, il y avait un ouvrage en chinois archaïque, mais que celui-ci aurait disparu lors d'un incendie. Est-ce vrai?

Hakuseki : C'est ce que raconte le peuple...

CHŏNgP'yŏNg : Si un tel ouvrage existe et qu'on ne le met pas à disposition, cela revient à dire qu'il n'existe pas. En effet, si cet ouvrage existe, c'est que le ciel en a décidé ainsi. Je ne pense pas qu'il y ait un sens quelconque à le cacher secrètement. N'en a-t-on pas fait une copie pour le transmettre?

Hakuseki : Il y a sans doute, dans le palais d'Atsuta de la province d'Owari, deux ou trois volumes sur tablette de bambous laqués, de nombreux lettrés y ayant servi. Ils sont probablement écrits en chinois archaïque.

NAMgang : Est-ce que vous ne pourriez pas nous les montrer sur le chemin du retour?

Hakuseki : Ceux qui sont en charge du palais les cachent. Je ne peux malheureusement rien y faire.

P'yongchŏN : Cai Zhonglang ${ }^{10}$ ayant caché le Lunheng ${ }^{11}$, [personne] n'a pu voir l'intérêt de cet ouvrage. [On a donc continué] de croire aux superstitions et on s'est comporté comme des [barbares] de Chu et Yue ${ }^{12}$. S'il existe un ouvrage mais qu'on ne le montre pas, c'est comme si cet ouvrage, finalement, n'existait pas.

Hakuseki : Lorsqu'on évoque l'Histoire des Zhou, on pense à un ouvrage d'une centaine de chapitres, sur les Trois Augustes et les Cinq Empereurs; le récit, attribué à Confucius, commence par la description des empereurs Yao et Shun et se termine par l'histoire des Zhou. Après les autodafés de la période Qin, les Han ont eu connaissance de l'existence du Classique de l'Histoire ${ }^{13}$ grâce à Fusheng ${ }^{14}$. Plus tard, on a retrouvé, caché dans le mur de l'ancienne demeure de Confucius, un exemplaire en caractères anciens. Si on ajoute à ceux-là les chapitres de l'ouvrage de Fusheng, on arrive à un total de cinquante-neuf chapitres. Mais selon les lettrés confucianistes chinois, l'ouvrage en caractères anciens daterait de la période des Jin orientaux (317-420) en raison de son harmonie générale. Des gens pouvaient lire un texte tel que celui de Fusheng. Mais quand on a trouvé celui caché dans le mur, personne n'était en mesure de le comprendre car il était écrit en caractères archaïques. Mais nous ne sommes plus au temps des Han! [A fortiori] y a-t-il aujourd'hui des gens qui soient capables de lire ce genre de texte? Si on veut comprendre la voie des Deux empereurs et des trois Rois, à quoi bon consulter un texte antérieur au Qin en caractères archaïques alors qu'il suffit de lire le texte en écriture moderne? Voyons, s'agissant de la voie des Deux empereurs et des trois Rois, le peuple aussi a son mot à dire. Nos ancêtres ont jadis caché [le texte ancien dans un temple]. Depuis ce temps-là, les gens le vénèrent. Si nous rendons impur le temple, [en pénétrant dans le lieu où le texte est conservé], le peuple sera offensé et nous risquons de nous attirer [leur colère]. Et puis, ce n'est pas parce qu'on ne peut pas vous montrer de texte sur les Deux empereurs et sur les trois Rois qu'on ne connaît pas la voie des Deux empereurs et des trois Rois. Il ne faudrait pas tomber dans [ce travers] ridicule.

Hakuseki : Ayant reçu l'ordre officiel de vous rendre [au Japon], vous avez franchi la mer pour venir jusque [chez nous] et vous avez surmonté beaucoup de difficultés 
pour le bien de nos deux pays. N'est-ce pas formidable? Quant à moi, [j'ai l'impression] d'avoir passé toute ma jeunesse au fond d'un puits car je n'ai jamais eu l'occasion de voir la mer, mais [ensuite] devenu adulte, j'ai rencontré deux ou trois représentants de votre pays en 1682. Plus tard, j'ai rencontré des Chinois, [des représentants] des Ryūkyū, des habitants de pays de l'Atlantique, d'Europe, tels que des Hollandais, des Suédois ou des Italiens qui sont venus [chez nous]. Et maintenant, je passe la journée à m'entretenir avec vous. C'est comme si j'avais reçu le privilège de faire le tour du monde.

cHŏngp'yŏng : Est-ce que le mot «Atlantique » désigne les pays qui sont à l'Ouest? Où se trouvent les pays comme la Hollande ou l'Italie?

Hakuseki : N'avez-vous pas de mappemonde dans votre pays?

NAMgANg : Nous avons des cartes anciennes sur lesquels ne figurent pas ces pays.

Hakuseki : L'Occident se trouve à l'ouest de l'Inde. Après avoir franchi une très grande distance, on trouve ce qui est connu sous le nom de grand et petit Occident. J'ai chez moi une carte. Je pourrai vous la montrer si vous voulez.

NAMgang : En effet, si vous avez une chose aussi précieuse, montrez-la-nous sans réserve.

Hakuseki : Je crains malheureusement que vous ne soyez pas en mesure de lire cette carte car tout est écrit dans la langue [vulgaire] de notre pays. Toutefois vous trouverez la même chose dans les ouvrages tels que le Yueling guangyi ${ }^{15}$ ou le Tongshubiang ${ }^{16}$.

NAMgang : Nous n'avons pas ce genre d'ouvrage.

Le lendemain, Hakuseki vint nous voir avec une petite carte et déclara :

Il y a deux sortes de mappemonde, les globes et les cartes. Elles sont annotées avec des caractères étrangers minuscules. On y trouve une description complète: le nom des lieux, des personnages, des traditions et des produits cultivés ou fabriqués, comme dans la carte d'environ un mètre trente de largeur du prêtre $\mathrm{Ri}^{17}$; on en trouve une reproduction dans le Yueling Guangyi, le Tianjing huo wen ${ }^{18}$ ou le Tushu Bian. On y a traduit la plupart [des indications] en écriture chinoise. J'ai fait reproduire cette carte en format réduit à Nagasaki. La méthode [des copistes] étant très précise, on arrive à faire des reproductions très fidèles. Il y a plusieurs centaines de noms de localités qui y sont mentionnés. Il faudra que vous fassiez traduire [la carte] dans votre langue, sinon vous ne pourrez pas la comprendre. Il y a sans doute des traducteurs à Tsushima qui pourront vous faire ça. Cette carte est une reproduction d'une des mappemondes offertes par des pays comme la Hollande et qui sont soigneusement conservées par notre administration. Je regrette de ne pouvoir vous montrer qu'une seule [de ces cartes]. (Notes de l'auteur ${ }^{19}$ )

CHŏngP'yŏng : Faut-il parcourir environ un millier de li20 pour se rendre dans les Ryūkyū? Quelle est la distance entre Fujian et Nagasaki?

Hakuseki : Selon nos modes de calcul, il y a cinq cents $l i$ [entre le Japon et les Ryūkyū]. Ces terres se trouvent dans la mer méridionale près de l'équateur. Pour cette raison, il y fait très chaud. Une même distance environ sépare Fujian de Nagasaki.

CHŏngp'yŏng : J'ai entendu dire que la route de Fujian est un nid de pirates. Les navires marchands n'y sont-ils pas attaqués?

Hakuseki : S'agit-il des pirates qui habitent le long de la rivière Min dans le Fujian? Je n'en ai pas encore entendu parler. 
NAMgANg : Il paraît qu'il y a un nombre fixe de navires qui font l'aller-retour [entre Nagasaki et Fujian], est-ce vrai ?

Hakuseki : Ce sont des navires qui viennent de Tangshan ou de la mer du Sud-Ouest. Chaque année, il en vient entre 160 à 170. Ils viennent sans encombre jusqu'au port de Nagasaki.

P'yongchŏN : On dit qu'il y a, depuis ces dernières années, des routes différentes, que les navires chinois ne se rendent plus [au Japon]. Qu'en est-il ?

Hakuseki : L'année dernière, un navire est venu de Nankin, mais il s'est perdu. Par la suite, on a vu apparaître des navires pirates en provenance du Zhejiang. Cette année, au printemps, une expédition militaire ${ }^{21}$ a été envoyée pour les punir et ramener leurs têtes. La navigation peut se faire sans problème. Et on peut aller [et venir] comme autrefois.

NAMgANg : De quels pirates parlez-vous? Et comment ont-ils disparu?

Hakuseki, sortant de sa poche un petit fascicule qu'il montre aux envoyés: Tei Jinshin, Chin Meiryu, Ri Rōryū ${ }^{22}$. Ils sont maintenant sous la garde des soldats de Nankin. Rōryū, ça correspond bien à un nom de bandit. Mais prendre Jinshin comme nom de pirate, il y a là vraiment de quoi rire ${ }^{23}$ !

NAMgang : Ce Tei Jinshin ne serait-il pas le rejeton de Tei Kin'yo ?

Hakuseki : C'est exact.

CHŏngP'yŏng : J'ai entendu dire que Li Madou [Matteo Ricci] était venu de son pays jusqu'ici en passant par Calicut et que ses écrits avaient été diffusés?

Hakuseki : Je n'ai qu'un volume du Jiaoyou lun ${ }^{24}$. La religion chrétienne étant rigoureusement interdite [dans notre pays], nous avons brûlé tous les livres ayant trait [à cette religion]. Le [Jiaoyou lun] est reproduit dans une anthologie qui regroupe des œuvres telles que le Baichuan xuehai et le Shuo fu ${ }^{25}$.

NAMgang: On dit que des envoyés des Ryūkyū viennent [au Japon]. Quels costumes portent-ils et quelle écriture utilisent-ils?

Hakuseki : Les coiffes et les habits des envoyés du Chūzan ${ }^{26}$ sont conformes à ceux de la période Ming. Généralement, ils portent un habit en soie d'une seule couleur. C'est la règle pour tous ceux qui sont d'un rang inférieur à celui de prince. Mais ils ont aussi des habits de brocard, violet, jaune, rouge, bleu et vert. Quant aux enfants, ils ont sur la tête des broches en or en forme de fleur et sont vêtus de vêtements à manches longues et amples. Ils nouent une grande ceinture autour des reins. Ils respectent aussi le système des rangs et des classes pour les fonctionnaires. On utilise dans ce royaume la même écriture que dans notre pays. Il y a [même] des gens qui maîtrisaient le waka. Dès le début de la période Ming, ils ont envoyé des ambassades en Chine. Les personnes versées dans les lettres chinoises étaient choisies comme interprètes et ambassadeurs. Par la suite, au début $d u \mathrm{XV}^{\mathrm{e}}$ siècle $^{27}$, la Chine a envoyé un grand nombre d'hommes originaire de la région du Fujian ${ }^{28}$. Le premier roi de Chūzan, Shun ten.ō, serait le fils du général Minamoto no Tametomo ${ }^{29}$. Par conséquent, il a gardé le nom de Minamoto. Son nom de clan est shō et il porte le titre de roi comme son ancêtre.

Hakuseki : Maintenant, tous les pays ont adopté la tenue vestimentaire de la dynastie des Qing. Votre pays garde les traditions de la dynastie des Ming ${ }^{30}$. Quel sens donner à tout cela? 
P'Yongchŏn : Tous les pays ont [adopté] des traditions barbares ${ }^{31}$. Seul le nôtre a conservé les préceptes de la civilisation. Les Qing nous considèrent comme des gens très policés et nous faisons en sorte de le rester. Sous le ciel, nous sommes les seuls Zhou de l'Est. Dans votre pays, désire-t-on suivre les préceptes de la civilisation ou non ? J'ai pu observer la qualité et la profondeur de l'enseignement [au Japon]. J'espère donc que vous parviendrez à changer vos mœurs.

Hakuseki : J'ai autrefois étudié le Livre des Odes. On raconte dans le chapitre des Odes et des Hymnes qu'un homme de Yin se rendit chez les Zhou en portant des habits d'autrefois. Au début, quand j'ai appris que vous alliez venir, j'étais impatient [de vous rencontrer], car on dit que vous êtes des descendants des $\mathrm{Yin}^{32}$. Les rites s'acquièrent dès la naissance, par conséquent on s'attendrait à ce que vous suiviez les préceptes des Yin. Ainsi, j'ai attendu votre venue dans notre pays afin de voir vos coiffes, vos tuniques ou vos tablettes. Mais je constate en fait qu'ils ne rappellent que la tenue vestimentaire des Ming, et qu'ils sont loin de faire penser à ceux des Yin ${ }^{33}$. Aujourd'hui, la dynastie Qing règne en [Chine] et les choses ont bien changé. Elle applique ses règles en se conformant à ses traditions. Votre pays, tout comme le royaume des Ryūkyū, se tourne vers le nord en tant que pays tributaire. Mais vous êtes autorisés à ne pas porter des nattes ou des vêtements [à la manière des Qing]. Est-ce que cela signifie que les Qing sont d'une grande magnanimité ? Ou bien, n'estce pas plutôt que vos deux pays puisent leur énergie de l'est [c'est-à-dire du Japon] ?

CHŏNgP'yŏng : J'ai entendu dire que votre pays connaît depuis longtemps les fusils et les sabres. J'ai déjà dit que je souhaitais voir votre technique militaire. Si vous souhaitez voir notre technique du tir à l'arc et notre art équestre, nous vous le montrerons avec plaisir.

Hakuseki : J'ai déjà entendu cette requête, et vous refaites aujourd'hui la même demande. C'est à croire que vous considérez depuis longtemps notre pays comme un pays de guerriers. Toutefois, écoutez bien ce que je vais vous dire. Il est vrai que l'art militaire est respecté dans notre pays. Dans le chapitre consacré au Gu, Yao dit clairement : " il y a le sacré (sei), le divin (kami), le guerrier (bu) et les lettres (bun). On ne doit pas accorder d'importance uniquement aux lettres et à ce qui est guerrier. » Quand notre monde a vu le jour, le divin et le sacré ont dominé et la vertu à laquelle tout le monde se conforme s'est répandue dans toutes les contrées. Lorsque [notre] empereur s'est trouvé affaibli, on a vu apparaître des chars de combat. À cette époque, le général Minamoto Yoritomo ${ }^{34}$ qui avait reçu du ciel courage et sagesse, a réprimé les rébellions par des actes héroïques sur les champs de bataille et a ainsi secouru la cour. Cela nous rappelle [les exploits] de Huan-Gong ${ }^{35}$ et de Wen-Gong ${ }^{36}$. On peut dire que depuis, nous avons perdu notre caractère paisible et sommes devenus des gens braves au tempérament solide. J'ai déjà eu l'occasion de dire à ce sujet que notre pays rappelait celui des Zhou de l'Ouest sous le règne de Wen-Wang ${ }^{37}$ qui, grâce à sa force de caractère, soumit les deux Sud, ou bien à celui de l'empereur Qin, qui par sa bravoure réunit les huit provinces et changea les mœurs du peuple. Confucius a dit : «L'homme de bien est forcément courageux ${ }^{38}$. Il devait en être de même dans notre pays. Notre divin ancêtre ${ }^{39}$ ayant ordonné, la paix fut rétablie par les armes mais il gouverna par les lettres. Et depuis un siècle, sa politique fondée sur quatre principes, le respect des lettres, des armes, de la fidélité et de la piété, est maintenue. C'est comme si [un homme ayant régné pendant plus d'un siècle] était capable de réprimer les violences et d'appliquer les justes sanctions ${ }^{40}$. J'ai entendu dire qu'autrefois, interrogé par le roi Sŏngjong Kangjŏng sur ses dernières volontés, Sin Munchung ${ }^{41}$ aurait dit avant de mourir, à propos de notre pays : "Surtout, n'oubliez pas la concorde avec le Japon ». Il a prononcé une telle phrase alors que 
nous étions en conflit ouvert ${ }^{42}$. Si vous vous inquiétez du sort de votre pays, ne devezvous pas garder à l'esprit ces paroles pour le bonheur de nos deux pays?

P'YǒngchŏN : Je suis un descendant de Sin Munchung. II a en effet prononcé ces paroles alors qu'il agonisait, afin d'éviter les dissensions et rapprocher nos deux pays. L'empereur Ming ayant eu connaissance de ces paroles aurait fait en sorte de suivre ses recommandations. C'est une chance extrêmement appréciable pour les générations futures de nos deux pays.

Hakuseki : J'ai déjà dit que la relation de bon voisinage reposait sur la loyauté. Je suis surpris d'apprendre que vous êtes un descendant de Sin. C'est une des grandes vertus de cet homme que d'être venu [au Japon] parler de paix. Non seulement son action a été bénéfique pour le monde en général, mais elle doit aussi avoir des retombées positives sur ses descendants ${ }^{43}$.

CHŏNgp'YŏNg : J'ai toujours [pensé] que vous étiez [simplement] un peuple de guerriers. Mais j'ai été impressionné, en venant ici, par le niveau de culture [de votre pays]. Cela mérite beaucoup de louanges. Les paroles de Sin Munchung doivent [effectivement] rester gravées éternellement [dans nos mémoires]. Actuellement nos deux pays vivent en paix, et les relations sont naturellement bonnes. Alors pourquoi troubler cela à cause d'une divergence d'opinions? Dans le but de nous distraire, nous avons sans cesse demandé à voir votre technique [militaire], or vous nous avez beaucoup éclairés sur ce sujet. Nous avons extrêmement honte de [notre comportement].

Hakuseki : Les relations pacifiques entre nos deux pays doivent reposer sur les principes du respect et de la sincérité. Vous êtes venus jusqu'ici en passant par Tsushima. Nos deux pays sont proches et nous nous connaissons relativement bien. Il ne faut pas oublier cette chance que nous avons.

P'YŏNgchŏN : Vous avez raison. Seulement, votre pays ne pousse pas la sincérité aussi loin que le nôtre.

Hakuseki : Depuis les époques les plus reculées, il y a des hommes qui aiment l'art militaire et qui répandent la haine. Ils passent leur temps à lutter, ne s'entraident jamais et ils provoquent de nombreuses dissensions. Je me fais vieux et je crains de n'avoir plus beaucoup d'années à vivre. De retour dans votre pays, transmettez sans faute à la cour l'importance de nos rapports afin que nous puissions renforcer les liens qui unissent nos deux pays, parlez-en ouvertement aux grands personnages du royaume.

CHŏngp'yŏng: N'est-ce pas un peu exagéré de parler de la sorte alors que nous ne pouvons pas dire quel est le degré de nos [rapports] ? Quoi qu'il en soit, nous savons qu'il y a une voie à suivre qui consiste à pérenniser les bonnes relations.

Hakuseki : Être inquiet et penser trop, tel est le lot d'un vieillard [comme moi]. Ne dit-on pas dans un poème: "On ne rejette pas un navet ou un radis parce que l'extrémité est un peu gâtée $»^{44}$ ? Alors, quoique sénile, je continue de parler et de faire des choix.

P'yŏngchŏn : Les Sō de Tsushima nous ont escortés jusqu'ici. Ils ont fait vraiment beaucoup d'efforts. Cela arrivera-t-il jusqu'aux oreilles de votre roi ?

Hakuseki : Il dirige avec la plus grande clairvoyance et rien ne lui échappe.

P'YŏngchŏN : Quelle est la règle dans votre pays concernant l'interdiction d'utiliser le nom du souverain? On ne doit pas souiller les deux noms [celui du souverain et celui de son père] mais dans votre pays, est-il de règle d'enfreindre cela? Les lettrés de votre pays, 
lorsqu'ils composent des poèmes, commettent ce genre d'outrage dans leurs compositions écrites. Est-ce à dire qu'ils ignorent [cet interdit] ?

Hakuseki : Nous utilisons une écriture ancienne, qui ressemble à la vôtre ${ }^{45}$, et depuis le Moyen Âge, le peuple a l'habitude de mélanger certains caractères chinois pour leur son [indifféremment de leur sens]. C'est devenu la norme d'écriture depuis longtemps. En fait, si nous pouvons déchiffrer les textes anciens, nous ne pouvons pas les lire à haute voix. Nous faisons en sorte de respecter l'interdit concernant les noms, toutefois, depuis quelque temps, ce n'est pas le cas.

P'YŏngchŏN : Il semble que certains termes utilisés dans la lettre officielle de réponse ne soient pas justes. Peut-on la voir avant [de l'accepter] ou bien devrons-nous attendre?

Hakuseki : Ce n'est pas moi qui ai écrit cette lettre. Je ne peux rien faire.

P'Yŏngchŏn : Connaissez-vous la coiffe que je porte?

Hakuseki : Non.

P'YŏNgchŏN : C'est un bokkin (jap. fukukin) ${ }^{46}$.

Hakuseki : Nous produisons des fukukin modernes mais je n'en ai jamais vus de style ancien. Pourriez-vous m'en donner un, comme ça je le ferai copier.

P'yŏngchŏn lui tend le sien.

Hakuseki : Je reçois cela comme si c'était un présent d'un ami.

P'YŏNgchŏN : Si vous souhaitez copier le bokkin, il vaut mieux commencer par étudier le chi kwan 緇冠. Vous trouverez la façon de le confectionner dans le Kalye toshik ${ }^{47}$.

Hakuseki : Ce que porte le vice ambassadeur et l'attaché ressemble à notre kinshuku. J'ai eu l'occasion de me rendre à Kyōtō l'année dernière: j'ai pu voir le style vestimentaire de la cour et j'ai pu me rendre compte qu'il remontait à un lointain passé $^{48}$. Au Japon, il est courant de voir des styles de vêtement qui remontent à l'époque des trois dynasties. Ainsi, ce que je porte ressemble au style des Zhou. Et le tan. $i^{49}$ a été fait en suivant des indications du Liking. Les confucianistes des Han et des Tang ont déjà largement expliqué tout cela.

NAMgaNg : Le style shimŭi a été fixé par Sima ${ }^{50}$. N'avez-vous pas d'autres ouvrages?

Hakuseki : Nous consultons le Liking car les confucianistes des Han et des Tang ont tout expliqué en détail. Pourquoi ces indications ne suffiraient-elles pas? Ce que nous appelons des habits de style Wu rappelle tout à fait le shimŭi. La différence est bien mince.

NAMgang : Vous ne consultez pas les Wengong Jiali/i, pour ce qui est des rites de l'adulte, du mariage, des funérailles ou des fêtes commémoratives?

Hakuseki : Dans notre pays, il y a cinq rites $^{52}$. Ils sont fondés en grande partie sur le code des trois dynasties. Ainsi, nous respectons les pratiques de Shaolian et Dalian qui auraient, selon Confucius, montré le modèle à suivre en ce qui concerne le deuil ${ }^{53}$. À l'époque des Tang, Lu Deming, dans ses commentaires du Zhouli ${ }^{54}$, fait référence à une explication de maître $Z_{\text {heng }}^{55}$ selon laquelle ces pratiques remonteraient à une époque très ancienne. Mais ces derniers temps, la plupart des confucianistes se conforment au précepte de Zhu Xi pour les rites de la famille.

Dans le Jingdian shiwen ${ }^{56}$ ou dans un des chapitres intitulé "Responsable des Divinations auprès du Supérieur des cérémonies sacrées du ministère du Printemps » des Commentaires du Zhouli, l'auteur Lu Deming mentionne qu'il y a neuf marques de respect ${ }^{57}$, telles que toucher le sol de son front ou bien claquer des mains. Et il ajoute le commentaire suivant: ces pratiques 
datent, comme l'indiquent les signes, de l'époque de maître Li, mais elles sont tombées en désuétude. Aujourd'hui, les Japonais [continuent] de saluer respectueusement en claquant les mains, mais si l'on en croit les explications de maître Zheng, cette pratique remonterait à des temps très anciens. (Notes de l'auteur)

Le rédacteur et trois secrétaires se joignent à la discussion.

Hakuseki, s'adressant au chargé de compilation: Avez-vous des enfants et quel âge ont-ils?

ToNggwAK $^{58}$ : J'ai un fils qui se prénomme Younjo. II a vingt et un ans.

Hakuseki : Chŏng est-il un descendant de Mongju ${ }^{59}$ ?

ToNggwaK : En fait, c'est un descendant de maître $P^{\prime} \mathrm{O}$ Ŭn${ }^{60}$ à la onzième génération. Ayant réussi le concours militaire, [P'o Ŭn ?] est devenu vice général dans une province. Au début du règne de la dynastie des Ming et du règne de Taejo, maître P'o Ŭn a fait partie de l'ambassade de félicitations qui s'est rendue en Chine et il a reçu de nombreux présents ${ }^{67}$. N'est-ce pas remarquable! Peu de personnes ont eu cette chance. P'o Ǔn en fait le récit détaillé dans ses écrits.

Hakuseki : Comment se fait-il que Chŏng ait passé le concours militaire?

ToNggwak : En fait, il était doué aussi bien pour les armes et que pour les lettres. Mais il a été invité par la Cour à prendre le commandement de l'armée. II a fait preuve de beaucoup d'adresse. On devrait le faire général.

Hakuseki, s'adressant au secrétaire Hong Set'e : Lors de l'ambassade de 1682, j'ai eu l'occasion de rencontrer un certain Chang Rang-ja. Vous ne seriez pas un membre de sa famille ${ }^{62}$ ?

LE SECRÉTAIRE HONg : J'ai entendu dire qu'il s'était rendu [au Japon], mais nous n'avons pas de lien de parenté.

Hakuseki : Je souhaiterais avoir des informations sur votre famille, sur votre position. Avez-vous des frères ou des enfants? C'est valable pour vous aussi secrétaires Ŏm et Nam.

LE CLeRC CIVIL SECRÉtAIRE HONg : Mon patronyme est Hong, mon nom d'usage est Sunyŏn et un de mes noms de plume est Kyŏng Ho. Je suis né à Namyang. J'ai travaillé au bureau des rites et cérémonies ${ }^{63}$. Quant aux concours, j'ai réussi l'examen de licence ${ }^{64}$ en 1677, et j'ai réussi le concours des lettres classiques en 1705. J'ai cinquante-neuf ans. Donc, il ne me reste plus longtemps à vivre. J'ai eu un enfant, mais il y a quelques années, je l'ai perdu. Votre question me rappelle ce passé douloureux.

LE CLERC CIVIL SECRÉTAIRE ŏM : Mon patronyme est Ŏm, mon nom d'usage est Hanjung, mon surnom est Jajŏng. Je suis né dans le canton de Yŏngwŏl de la province de Kangwŏng. Mon nom de plume est Yŏngho ${ }^{65}$. J'ai quarante-huit ans. J'ai passé l'examen de licence en 1690 et j'ai été nommé docteur au Pisŏsŏng ${ }^{66}$ le troisième mois de la même année. Je suis devenu vice surveillant à la Cour des Dépêches ${ }^{67}$, j'ai ensuite été gouverneur de la province de Kochang et je viens en tant que vice secrétaire. J'ai un seul frère, Ŭnghaeng, du nom de Hannyŏn. J'ai deux fils, l'aîné s'appelle Kyŏng, le cadet Bang.

LE CLeRC CIVIL SeCRÉtAire sŏngJung: Mon nom patronymique est Sŏngjung. Je suis originaire du comté de Ǔiryŏng et je me suis rendu à la capitale. Je suis le troisième fils de maître Hogok ${ }^{68}$ qui a été Rédacteur en 1655. J'ai quarante-sept ans. J'avais un grand frère du nom de Jŏngjung. II était inspecteur ${ }^{69}$ dans la province de Kyŏngsan, mais il est décédé en 1704. II me reste un grand frère et un petit frère mais ils n'occupent aucune fonction.

Hakuseki : Le rédacteur officiel Sŏngwan ${ }^{70}$ [qui est venu au Japon] en 1682 est-il en bonne santé? 
LE CLERC CIVIL SECRÉTAIRE Hong : Il est décédé cet été.

Hakuseki : Il a franchi la mer pour nouer des relations amicales. Quelle tristesse d'apprendre qu'il est six pieds sous terre!

ToNggwaK : II n'avait pas une charge élevée, et il n'a pas eu une longue vie. C'est vraiment triste!

Hakuseki : Ch'uihŏ ${ }^{71}$ a-t-il eu des enfants ?

TONggwaK : Il a eu deux enfants.

Hakuseki : C'est fort bien! Quand vous serez de retour dans votre pays, transmettezleur mes hommages.

TONggWAK : Il en sera fait ainsi.

Hakuseki : Aujourd'hui, je rencontre un descendant éloigné de P'o Ǔn Chŏng, comme c'est étrange. Autrefois, en l'an deux de l'ère Eiwa, autrement dit en l'an dix de l'ère Hongwu fondateur de la dynastie Ming (1377), Chŏng est venu [au Japon] en tant que représentant de Koryŏ, et il a rencontré l'envoyé de Kyūshū [Kyūshū setsudo shi], Minamoto no Sadayo ${ }^{72}$, pour parler de la paix entre les deux pays. Au début de la dynastie Chosŏn, Pak Kongton est venu [dans notre pays] afin de rétablir les anciennes relations qui avaient été établies à l'époque de Koryŏ. Lorsque vous êtes arrivés [à Edo ?] vous avez été accueillis par un Minamoto ${ }^{73}$, un descendant à la neuvième génération de Sadayo.

CHŏNgP'yŏNg : Chŏng Chan-sul74 est un descendant à la onzième génération de P'o Ŭn. Et il devrait y avoir encore de nombreux [descendants] de grande qualité qui donneront de bons soldats. II fait partie [de l'ambassade]. Il est alors vraiment curieux qu'on rencontre un descendant de Minamoto. Je ne connais pas encore son patronyme et j'aimerais avoir des informations sur lui. Est-il venu dans le bâtiment d'accueil [pour nous voir] ?

Hakuseki : Le descendant de Minamoto, envoyé de Kyūshū, a pour patronyme I. Il a le grade de konno shōshō. Il est aussi gouverneur de la province de Buzen. Il est connu généralement sous le nom de Shinagawa. Le jour où vous êtes arrivés ici pour la première fois, il est venu à votre rencontre. Au moment de votre départ, il ira, probablement, vous saluer respectueusement.

CHŏNgP'yŏNg : La rencontre d'aujourd'hui marque une étape importante dans les relations futures de nos deux pays. Il faut par conséquent consigner [notre conversation] pour en avoir une trace dans nos deux pays.

Hakuseki : Autrefois, le seigneur Chŏng est venu [au Japon] pour établir la paix entre nos deux pays, puis ce fut le tour du seigneur Sin. Plus récemment, le seigneur Hogok Nam est venu en 1655. Maintenant, je parle avec son excellence cho et j'apprends qu'il est un descendant éloigné du seigneur Sin. Im et $\mathrm{Yi}^{75}$, vous aussi vous suivez les traces de Chŏng et de Nam. D'ailleurs, je découvre également que maître Yi est également un disciple du seigneur Nam. Comment se fait-il que vous seuls formiez une telle lignée de gens vertueux ? Il y a autrement dit une grande continuité. C'est en réalité une très grande joie pour nos pays. Dire que j'ai le bonheur d'assister à un tel événement qu'on ne peut voir qu'une seule fois dans toute sa vie! Vous devez noter [tout ce qui a été dit ici] pour le transmettre aux générations futures, et que [nos paroles] restent à jamais gravées.

LE SECRÉTAIRE NAM : II est extrêmement rare d'avoir l'occasion d'aller à l'étranger [pour parler] tel que nous l'avons fait aujourd'hui. C'est comme si deux mondes s'étaient 
rencontrés. N'est-ce pas formidable ! [Une fois de retour en Corée], je tournerai mon regard vers l'est et à la vue des rayons de soleil, ma tristesse [suite à la séparation] s'estompera.

chŏngp'yŏng: On peut lire dans les classiques «Aussitôt que nous nous sommes rencontrés, nous avons ri comme de bons amis. Qui peut dire ce qui nous divise ? " ${ }^{76}$. Au cours de la réunion d'aujourd'hui, nous avons plaisanté. C'est une chose bien rare depuis que nos deux pays entretiennent des relations. Si les esprits s'entendent, on peut oublier complètement les divisions ${ }^{77}$.

P'YŏNgchŏN : Il y a bien des limites géographiques. La mer et la terre nous éloignent. Une fois que nous serons séparés, nous n'aurons plus l'occasion de nous reparler. Comment ne pas être triste [au souvenir] d'une telle conversation?

Mais un croissant de lune brillant peut atteindre un cœur distant de mille lieux et l'éclairer ${ }^{78}$.

Hakuseki : Je suis triste. On peut lire à la fin du poème Xi Sang «Je garde son souvenir au fond de mon âme; ne pourrais-je jamais l'oublier? " ${ }^{79}$. Lorsque vous serez de retour dans votre pays, ayez une pensée [pour moi] en regardant vers l'est.

Et d'ajouter: Nous devons consigner le précieux entretien que nous avons eu aujourd'hui. Je vous serais reconnaissant de bien vouloir m'envoyer prochainement les différentes notes qui ont été prises.

Il se retire après avoir présenté ses salutations.

Fin de la conversation.

\section{NOTES}

1. Cho T'ae-ŏk 趙泰億 (1675-1728) portait le titre de T'ongjŏng Taebu Ijo Ch'amŭi Chijekyo 通政大夫 吏曹参議知製教. Il est difficile de traduire en français les titres des Coréens car des équivalents en langue occidentale n'ont pas toujours été fixés. Nous avons donc préféré raccourcir le titre de l'ambassadeur dans la traduction en nous référant à une traduction de Maurice Courant dans son «Répertoire historique de l'administration coréenne », in Cahiers d'études coréennes, $\mathrm{n}^{\circ} 3$, Collège de France, 1986. Keith Pratt et Richard Rutt, quant à eux, traduisent taebu par "grand personnage " dans le Korean History and Cultural Dictionnary. Le titre taebu était accompagné d'un préfixe attributif de deux caractères, comme c'est le cas pour l'ambassadeur, qui est donc un grand personnage en charge des affaires courantes (T'ongjŏng 通政).

2. Alias Cho T'ae-ŏk.

3. Alias Yi Bang-on 李邦彦. Il a le titre d'attaché (chongsagwan 従事官).

4. Ouyang Xiu 歐陽修 (1007-1072) : lettré chinois, historien, philosophe, poète, ayant relaté dans l'un de ses ouvrages (日本刀歌) l'histoire de Xu Fu 徐福, dont il est question ci-dessous.

5. Dans son propos, Hakuseki distingue le Daijinbyō 大神廟 du Taisha, ou O-yashiro 大社.

6. Guwen Shanshu 古文尚書, Le Classique de l'Histoire.

7. Alias Im Su-gan 任守幹, vice ambassadeur, auteur du Tongsa ilgi 東槎日記, dans lequel on trouve une autre version du Kōkan hitsudan.

8. Katosho 科斗書: écriture chinoise archaïque. Il s'agit de graphies sigillaires imitant plus ou moins la forme des têtards (kato).

9. $\mathrm{Xu} \mathrm{Fu}$ 徐福: Alchimiste légendaire qui aurait vécu sous le règne du premier empereur chinois Shihuangdi (259-210). Il serait parti à la recherche d'un élixir qui empêche le vieillissement et donne l'immortalité. Il se serait rendu dans différentes régions du Japon, emportant avec lui divers textes chinois, dont une version en écriture ancienne du Shujing 書経 ou Shanshu 尚書. Il 
les aurait ainsi sauvés de l'autodafé, car à cette époque, en Chine, le Premier ministre Li Si 李斯 (280-208) avait ordonné qu'on brûlât les textes historiques (214 av. J.-C.) et les textes littéraires, dont de nombreux ouvrages confucéens (213 av. J.-C.).

10. Cai Zhonglang 蔡中郎 est le titre donné à Cai Yong 蔡隹 (133-192), "Cai le Général commandant les gardes du palais ». Cai Yong a établi le texte des six livres classiques, gravé sur stèles en 175 à l'académie de Luoyang.

11. Lunheng 論衡 : ouvrage écrit en 83 par Wang Chong (27-97), et consacré à la critique des superstitions de son époque.

12. Les Chu 楚 et les Yue 越 étaient réputés avoir des coutumes barbares.

13. À l'époque des Han, les Classiques « modernes » désignaient les versions officielles transmises en écriture cléricale courante au début des Han après l'uniformisation des graphies sous les Qin. Voir Anne Cheng, Histoire de la Pensée Chinoise, Folio, 1997.

14. Fu Sheng 伏生 (264 ?-170). Lorsque sous les Qin (221-206), on a commencé à brûler les textes confucéens, Fu Sheng en aurait caché une partie. Par la suite, sous les Han, il les a redécouverts, mais seuls vingt-huit chapitres étaient lisibles. Des recherches ont été menées pour récupérer les textes perdus mais sans succès. Fu Sheng a donc transmis oralement ce qu'il connaissait des textes disparus à un de ses disciples. Les vingt-huit chapitres et le texte transmis oralement ont formé par la suite le Jinwen Shangshu.

15. 月令廣義: «Commentaires sur les ordonnances mensuelles », l'un des chapitres du Livre des Rites.

16. 圖書編: Bibliographie encyclopédique en cent vingt-sept volumes de l'ère Wanli, des Ming, où les ouvrages sont rangés par matières.

17. Matteo Ricci (1552-1610), désigné sous le nom de Ri sanjin 利山人 dans le texte d'Arai Hakuseki, le nom chinois de Matteo Ricci étant Li Madou 利瑪䆩. Missionnaire jésuite italien, mathématicien et astronome, il s'est rendu en Chine en 1583. En 1601, il rencontre l'empereur Wanli.

18. 天經或問 : Traité d'astronomie chinois de You Ziliu 遊子六, importé au Japon et publié en l'an 15 de l'ère Kyōhō (1730).

19. Notes ajoutées lors de la compilation par Cho T’ae-ŏk.

20. Un li correspond à un peu moins de quatre kilomètres.

21. Sans doute chinoise.

22. N'ayant pas d'information sur le pays d'origine de ces pirates, nous avons choisi de citer leur nom dans une lecture japonaise.

23. Sans doute en raison des idéogrammes que le pirate s'est attribué et dont le sens est « de tout son cœur ».

24. 交友論: Traité sur l'amitié, 1595.

25. Le Baichuan xuehai 百川學海 est une collection compilée par Zuo Gui, de la dynastie Song, ère Xianchun (1265-1274). L'auteur y a recueilli des anecdotes et histoires non officielles des Tang et des Song. Le Shuo fu 説郛 est un recueil de récits courts et de notes d'écrivains antérieurs, rédigé par le lettré chinois Tao Zongyi 陶宗儀 ( ?-1369).

26. Trois royaumes rivaux se sont formés au cours du XIV siècle à Okinawa : Chūzan 中山, Hokuzan 北山 et Namzan 南山. Le roi de Chūzan, connu sous le nom de Satto 察渡, a été le premier à envoyer un tribut à la Chine des Ming. Il a aussi fait construire le château de Shuri.

27. Entre 1403 et 1424 pour être plus précis.

28. Durant la dynastie des Ming, ces hommes, connus sous le nom de Binjin 閩人, avaient été envoyés par l'empereur chinois dans les Ryūkyū, sous la demande pressante du roi Satto qui, en raison de l'intensification du commerce de transit avec la Chine, réclamait des personnes sachant parler le chinois.

29. Selon une légende, Shun ten.ō serait le fils de Minamoto no Tametomo 源爲朝 (1139-1170). 
30. Après le renversement de l'empire Ming en 1644, la cour coréenne a continué de garder des traditions héritées des Ming, contre les pratiques des Mandchous.

31. L'envoyé coréen utilise le terme sajin 左社, littéralement " porter le revers à gauche ", pour décrire les us barbares.

32. Les princes de Yin étaient des descendants de Kija, fondateur du premier royaume coréen.

33. Hakuseki mentionne deux coiffes de l'époque des Yin, le shōho 章甫, dont Confucius aurait fait usage, et le fuku 黼星 (chapeau brodé).

34. Premier shōgun.

35. 齊桓公 : premier Hégémon de Qi (période Chunqiu, de Printemps et Automne).

36. Jin Wen Gong 晋文公, ou duc de Wen de l'État de Jin.

37. Wen-wang 文王 (1148-1051 av. J.-C.), souverain vertueux de Zhou. Les Zhou étaient, semble-til, plus guerriers que leurs ennemis, les Yin, d'où la comparaison.

38. Voir le chapitre quatorze, Kenmon 憲問, des Analectes de Confucius：「子日。有徳者必有 言。有言者不必有徳。仁者必有勇。勇者不必有仁」. «Le maître dit : L'homme de vertu parle forcément correctement, mais ceux qui parlent correctement ne sont pas forcément vertueux. L'homme de bien est forcément courageux mais un homme courageux n'est pas toujours un homme de bien."

39. Tokugawa Ieyasu.

40. Hakuseki fait référence aux Analectes, chapitre treize, Shiro 子路：「子日、善人爲邦百年、 亦可以勝残去殺矣、誠哉是言也」. 《Le maître dit : Si des hommes devaient gouverner durant plus d'un siècle, ils seraient capables de réprimer les violences et d'appliquer les justes sanctions. Il n'y a rien de plus vrai. "

41. Il s'agit de Sin Suk-chu 申叔舟 (1417-1475) qui s'est rendu au Japon en 1443 en tant que membre d'une ambassade coréenne. Il a laissé un ouvrage célèbre rédigé en 1471, le Haedong jegukki 海東諸国紀, dans lequel il fait une description du Japon et des Ryūkyū.

42. En 1419, la Corée envoya une expédition punitive à Tsushima pour détruire les bases arrière des pirates dont elle subissait les assauts, mais elle décida dans le même temps d'ouvrir trois ports, au sud de la péninsule, pensant ainsi mettre fin à leurs activités en favorisant les échanges commerciaux avec le Japon. En 1510, les Japonais demeurant dans les trois ports se soulevèrent contre les autorités locales, ce qui entraîna la fermeture de ces mêmes ports jusqu'en 1572 .

43. Hakuseki fait référence à un adage tiré du Livre des Mutations 易経:「積善之家必有余慶」， «Si l'on fait beaucoup de bonnes œuvres, on reçoit du bonheur en retour. »

44. La citation, prononcée en chinois "caifeng caifei wuyi xiati ", est tirée du poème "Gufeng " (vent d'est), qui fait partie du livre III du Shijing (anciennement Cheu king: Canon de la poésie). Ce sont les plaintes d'une femme chassée par son mari et remplacée par une concubine.

45. L'alphabet coréen, le hangŭl. Sur la connaissance qu'avaient les lettrés d'Edo de l'écriture coréenne, voir l'article de François Macé : http://cipango.revues.org/1125.

46. 幅巾: coiffe officielle de couleur noire.

47. Ouvrage confucéen relatif au code vestimentaire.

48. Le code vestimentaire de la cour impériale japonaise, inspiré du code établi sous le règne du premier empereur de Chine Shi Huangdi 始皇帝, fut en vigueur jusqu'au début de la période Meiji.

49. Cor. Shimŭi. Selon Nakai Riken 中井履軒 (Tan.i zukai 深衣困解, 1765) ce style vestimentaire remonterait à l'antiquité chinoise et serait l'équivalent du fudan 燕服, un habit réservé aux nobles.

50. Il s'agit probablement de Sima Qian 司馬遷, historien chinois de la période Qin, auteur des Mémoires historiques (Shiji 史記).

51. 朱子家禮: Rites de la famille de Zhu Xi 朱喜.

52. Gorei 五礼: Kichi (ou kitsu)-rei 吉礼, kyōrei |凶礼, hinrei 賓礼, gunrei 軍礼, karei 嘉礼. 
53. Chapitre dix-huit du Liki 禮記：「孔子日 少連 大連 善居喪 三日不㤐 三月不解 期悲哀 三年 憂東夷之子也」. «Confucius dit : Shaolian et Dalian ont dignement porté le deuil. Pendant trois jours, ils ont consacré beaucoup d'attention au défunt, pendant trois mois, ils ont continué de porter l'habit de deuil, et pendant trois ans, ils n'ont cessé de pleurer le défunt... »

54. Lu Deming 陸徳明 (556-627), philologue sous la dynastie Tang.

55. Il s'agit probablement de Zheng Xuan 鄭立 (127-200), qui a laissé des commentaires du Livre des Odes, du Mémoire sur les bienséances (Yili), du Zhouli, du Liji, du Lunyu et du Shangshu.

56. 經典釋文: Explication de textes classiques et canoniques, de Lu Deming, exégèse consacrée aux textes confucéens et taoïstes (Laozi, Zhuangzi).

57. Dans le texte original : 一曰日稽首. 二日頓首. 三日空首. 四日振動. 五日吉拜. 六日凶 拜. 七日奇拜. 八曰褒拜. 九日肅拜」.

58. Yi Tonggwak 李東郭.

59. Il semblerait que Hakuseki parle d'un membre de l'ambassade, Chŏng Chan-sul 鄭絓垻述, un lettré de la seconde moitié de la période Chosŏn. Celui-ci était un fonctionnaire du sŏnjŏnkwan 宣 傳官, un bureau qui transmettait des ordres du roi sous la dynastie des Yi.

60. P’o ŭn 圃隱, alias Chŏng Mongju 鄭夢周 (1337-1392) : lettré, militaire et diplomate de la fin de la période Koryŏ qui s'est rendu en Chine et au Japon et qui a pris part aux combats contre les Jürchen et les pirates japonais. Rallié au Ming mais loyal à la dynastie Koryŏ, il s'opposa au fondateur de la dynastie Chosŏn et mourut assassiné. Aux yeux des Coréens, il représente la figure emblématique de l'inébranlable loyauté.

61. Il s'est rendu en Chine dans le cadre d'une ambassade exceptionnelle de félicitations, chinhasa 進賀使.

62. Hakuseki avait fait la connaissance de Hong Se-t'e 洪世泰 (1653-1725) en 1682. En 1711, Hakuseki pose cette question car le nom de plume de Hong Se-t'e est aussi Changrang 滄浪.

63. 太常待判官 : plutôt que le signe 待 il faut sans doute lire 寺 dans le texte original. Taesang 太 常 renvoie à une fonction tandis que p'ankwan 判官 renvoie à un rang.

64. Durant la période Chosŏn, l'examen de licence (chinsagwa) se déroulait en plusieurs épreuves : une composition de poème en chinois classique, un poème en vers, un texte en prose administratif et une composition sur un thème donné.

65. Hanjung 漢重 serait né en 1665.

66. 秘書省: l'un des six ministères, le système étant calqué sur le modèle de l'administration chinoise.

67. Surveillant à la Cour des dépêches : kyogŭm Sŭngmunwŏn 承文院校檢.

68. Nam Yong-ik 南龍翼 (1628 1692), dont le nom de plume était Hogok 壸谷, fut membre de l'ambassade coréenne qui se rendit au Japon en 1655, en qualité d'attaché chongsagwan.

69. Kwanch'alsa 観察使.

70. Sŏngwan 成琬 est un nom de plume: lettré de Songgyun'gwan 成均館, célèbre académie confucéenne publique fondée en 1398 à Séoul, cet homme avait fait partie de l'ambassade de 1682. Il avait apprécié le recueil de poèmes remis par Arai Hakuseki et il avait écrit une préface et une postface à ce recueil.

71. Ch'uihŏ 翠虛 alias Sŏngwan.

72. Minamoto no Ryōshun 源了俊 ou Imagawa Sadayo 今川貞世 (1326-1420).

73. Du grade de cour konno shōshō 近衛少将.

74. Membre de l'ambassade en tant que jaesulgwan 製述官 (spécialiste de la composition chinoise et de la poésie).

75. Im Su-gan alias Namgang dans le texte.

76. Citation tirée du Shiki, et qui fait aussi référence à Confucius.

77. Référence à Sōshi.

78. Le sens de cette phrase est obscur. Fait-elle allusion au fait que même une fois séparés, les grands esprits ne peuvent pas s'oublier? 
79. Shi jing, Livre II, Chant IV ( $\left.\mathrm{n}^{\circ} 228\right)$, Xi Sang. Le poète exprime son estime et son affection pour les hommes sages. Il les compare aux mûriers touffus qui croissent dans les terrains bas et humides.

\section{RÉSUMÉS}

Notes d'une «conversation au fil du pinceau» (hitsudan) entre Arai Hakuseki et des officiels coréens, à l'occasion de la visite à Edo de l'ambassade coréenne en 1711.

Notes from a "brush conversation" (hitsudan) between Arai Hakuseki and Korean officials, during a visit from the Korean delegation to Edo in 1711.

\section{INDEX}

Index chronologique : Edo (1603-1867)

Mots-clés : Arai Hakuseki (1657-1725), shogunat Tokugawa (1603-1867), hitsudan, Kōkan hitsudan, diplomatie

キーワード : Arai Hakuseki 新井白石 (1657-1725), Tokugawa bakufu 徳川幕府 (1603-1867), hitsudan 筆談, Kōkan hitsudan 江關筆談, gaikō外交, Edo 江戸, Kankoku 韓国, Edo jidai 江戸時 代 (1603-1867), rekishi 歴史

Index géographique : Edo, Corée

Thèmes : histoire

Keywords : Arai Hakuseki (1657-1725), Tokugawa shogunate (1603-1867), hitsudan, Kōkan hitsudan, Diplomacy, Edo, Korea, Edo Period (1603-1867), History 